\title{
Decreased Odor Avoidance after Electric Shock in Drosophila Mutants Biases Learning and Memory Tests
}

\author{
Thomas Préat \\ Institut Alfred Fessard, Centre National de la Recherche Scientifique, 91190 Gif-sur-Yvette, France
}

The Drosophila mutants amnesiac, dunce (dnc), and rutabaga were isolated after associative conditioning tests, during which animals were trained to associate the presence of an odor with that of electric shocks (ES). In the absence of conditioning, the odor avoidance (OA) of these mutants was shown to be normal, indicating that their poor associative conditioning performance was attributable to specific learning or memory deficits. However, I show that the OA of the mutants is greatly decreased after their exposure to ES. This effect can last for hours. These results strongly suggest that part of the defect displayed by these mutants in associative conditioning tests does not correspond to a learning or memory deficit but might arise from abnormal sensitivity to stressful stimuli. I looked at the OA after ES of two previously characterized dnc mutants. $D f(1) N^{79 f}$

Several mutations affecting associative learning and memory have been characterized in Drosophila, including dunce (dnc), rutabaga (rut), amnesiac (amn), and linotte (lio) (Dudai et al., 1976, 1984; Quinn et al., 1979; Dura et al., 1993). dnc and rut encode, respectively, a phosphodiesterase and an adenylate cyclase (Chen et al., 1986; Levin et al., 1992), two enzymes involved with the cAMP pathway. amn encodes a putative neuropeptide that might regulate adenylate cyclase activity (Feany and Quinn, 1995), and lio encodes a putative tyrosine kinase (Dura et al., 1995) involved in adult brain development (Simon et al., 1998). Despite recent progress (Zhong and Wu, 1991; Qiu and Davis, 1993; Dauwalder and Davis, 1995), the precise physiological roles of the Drosophila proteins Amn, Dnc, and Rut are not fully understood. In particular, although Dnc and Rut accumulate in the mushroom bodies (Nighorn et al., 1991; Han et al., 1992), an insect structure involved in learning and memory (Erber et al., 1980; Davis, 1993; Hammer, 1993; de Belle and Heisenberg, 1994), they are also expressed in other parts of adult brain (Nighorn et al., 1991; Han et al., 1992) in which their possible roles are unknown.

The conditioning protocols originally used to isolate and characterize most of Drosophila learning and memory mutants associate an odor with electric shocks (ES) (Quinn et al., 1974; Tully

\footnotetext{
Received May 21, 1998; revised Aug. 3, 1998; accepted Aug. 7, 1998.

This work was supported by the Human Frontier Science Organization, the Fondation pour la Recherche Médicale, the Association pour la Recherche contre le Cancer, and the Centre National de la Recherche Scientifique (ATIPE 7). Part of this work has been performed at the Universität Würzburg (Lehrstuhl für Genetik, Am Hubland, D-97074 Würzburg, Germany), and I thank researchers from that laboratory for their valuable criticisms of this work. I thank Ronald Davis for providing fly stocks and Jean-Maurice Dura, Yves Fregnac, Martin Heisenberg, Raphael Hitier, Lucy Vincent, and Jean-Didier Vincent for their helpful comments on a previous version of this manuscript.

Correspondence should be addressed to Dr. Thomas Préat, Institut Alfred Fessard, Centre National de la Recherche Scientifique, 91190 Gif-sur-Yvette, France. Copyright (ㄷ) 1998 Society for Neuroscience $0270-6474 / 98 / 188534-05 \$ 05.00 / 0$
}

specifically decreases Dnc expression in the mushroom bodies, leading to a normal level of learning but decreased memory. $D f(1) N^{79 f}$ mutants displayed a normal OA after ES. $D f(1) N^{64 j 15}$ affects the entire brain expression of Dnc, leading to decreased learning and memory. $D f(1) N^{64 j 15}$ animals showed a strong decrease of their OA after ES. Thus, the lack of Dnc "general" expression is most likely responsible for the OA defect, which would be responsible for the apparent learning defect after conditioning. In contrast, the Dnc phosphodiesterase accumulated in the mushroom bodies would be involved specifically in memory formation.

Key words: Drosophila melanogaster; learning and memory mutants; cAMP; stress sensitivity; odor avoidance; conditioning controls

and Quinn, 1985). Naturally, before the abnormal performance of the mutants could be linked to a learning or memory defect, it was shown that untrained mutants could react normally to the stimuli used for the conditioning and, in particular, that their odor avoidance (OA) was normal (Dudai et al., 1976, 1984; Quinn et al., 1979; Dura et al., 1993). However, the possibility that ES presentation during conditioning could itself affect odor perception was never explored. This issue is crucial when one needs to compare a putative learning or memory mutant with a reference wild-type stock, because the ES might differentially affect the two groups. Thus, to characterize a mutant deficient in associative learning or memory, it is essential to separate a bona fide learning or memory defect (related to the association of stimuli) from behavioral deficits merely related to abnormal perception of the stimuli after the conditioning treatment. I show here that the mutants amn, $d n c$, and rut display a very strong decrease of their OA after their exposure to ES. Two deficiencies affecting different sets of $d n c$ transcripts allowed the separation of memory defects from nonspecific deficits.

\section{MATERIALS AND METHODS}

All of the mutant stocks have a Canton-S background. This issue is important, because different wild-type backgrounds lead to different learning and memory aptitudes (Tully and Quinn, 1985). The three ethylmethane sulfonate mutations $a m n, d n c$, and rut were originally induced in a Canton-S background. The background of the two $\mathrm{P}$ mutants rut ${ }^{\mathrm{P} 2080}$ and lio $^{1}$ was "cantogenized" by outcrossing $w^{1118}$ $r u t^{\mathrm{P} 2080} / w^{1118}$ females and $w^{1118}$, lio ${ }^{1} /+$ females to $w^{1118} / Y$ (Canton-S) males for five generations. The $l i o^{2}$ mutant was cantogenized by outcrossing $w^{1188}$, lio ${ }^{1} /$ lio $^{2}$ females to $w^{1118} / Y$, lio $^{1} /$ lio $^{1}$ (Canton-S) males for five generations. The $w^{\mathrm{a}} D f(1) N^{79 \mathrm{f}} / w^{+} Y \times C D(1), y w f$ and $D f(1) N^{64 \mathrm{j} 15} /$ $w^{+} Y \times C D(1), y w f($ Canton-S) stocks were provided by Ronald Davis (Baylor College of Medicine, Houston, TX). A cantogenized $w^{\text {a }}$ $D f(1) N^{79 f} / w^{+} Y \times C D(1), y w f$ (Canton-S) stock was generated by outcrossing for nine generations $w^{\text {a }} D f(1) N^{79 f} / w^{1118}$ females to $w^{1118} / Y$ 

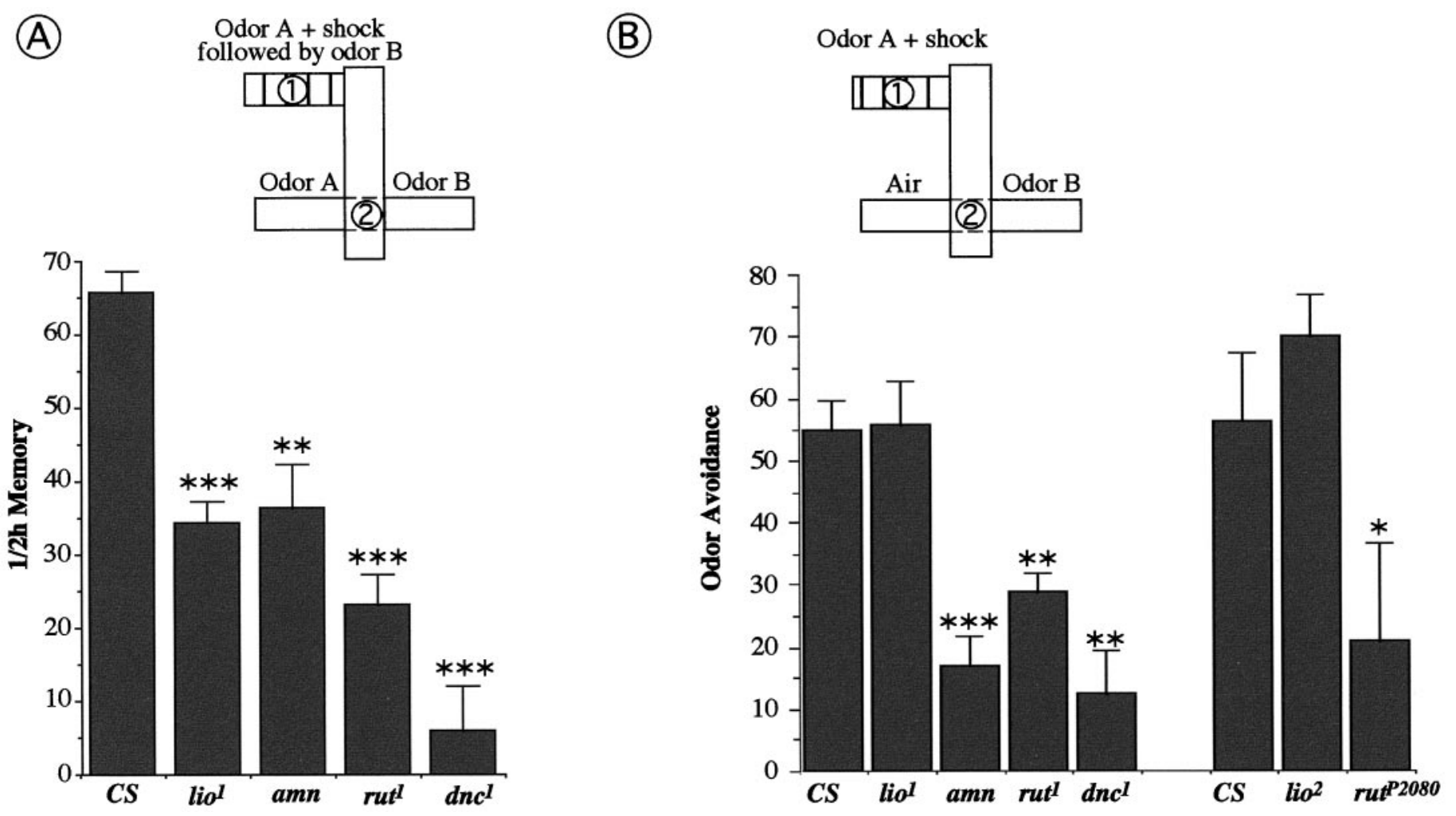

Figure 1. A, Half hour memory of normal [Canton-S $(C S)]$ and mutant stocks. Flies were trained in the grid tube (position 1) and tested at the choice point (position 2). Bars represent mean \pm SE performance index. These scores match previously published values (Tully and Quinn, 1985; Dura et al., 1993). ${ }^{* *} p<0.01$; *** $p<0.001$; one-way ANOVA. $n=4$ groups. B, OA of normal and mutant stocks after ES. OA to octanol (position 2) after presentation of ES combined with methylcyclohexanol (position 1) (see Materials and Methods). Left, CS, lio ${ }^{1}$, amn, rut ${ }^{1}$, and dnc ${ }^{1}, 120 \mathrm{~V}$ were used together with medium humidity. Right, $C S$, lio $^{2}$, and rut ${ }^{\mathrm{P} 2080}, 60 \mathrm{~V}$ were used with high humidity. Note the similar scores displayed by Canton-S under both conditions. This experiment was performed blind as to genotype. ${ }^{*} p<0.05 ;{ }^{* *} p<0.01$; ${ }^{* * *} p<0.001$; one-way ANOVA. $n=6$ groups.

(Canton-S) males. A $w^{1118} / w^{+} Y \times C D(1), y w f$ (Canton-S) stock was also generated.

For associative memory tests, flies were conditioned with the Pavlovian procedure developed by Tully and Quinn (1985), with a few adaptations. During training, groups of 50-100 flies were first exposed for $60 \mathrm{sec}$ to a first odor (odor A) (either undiluted 3-octanol or 4-methylcyclohexanol), during which time they received ES ( $1.5 \mathrm{sec}$ pulses of DC). After a $45 \mathrm{sec}$ rest period, flies were exposed for $60 \mathrm{sec}$ to the second odor (odor B), which was not paired with ES. Flies were then kept for 30 or $90 \mathrm{~min}$ in a vial with regular solid food. For memory testing, flies were transported to the choice point of a T maze, allowed to choose between the two odors for $120 \mathrm{sec}$, and counted. The performance index represents a normalized probability of correct choice. A score of 0 thus corresponds to a 50:50 distribution.

For OA tests, flies were treated in the upper chamber as for the associative conditioning, except that presentation of the second odor was omitted and replaced by exposure to air. For OA testing, treated animals were transported to the choice point of the T maze, allowed to choose between the new odor and air, and counted. A performance index was calculated as for associative conditioning. An index of 0 corresponds to a 50:50 distribution. An index of $100 \%$ corresponds to complete avoidance of the odor. Odors were used undiluted as by de Belle and Heisenberg (1994). Two groups of the same stock were run successively, and the side of the test tube with odor was alternated. To remove odor traces from the previous run before each new experiment and in the absence of flies, odor and fresh air, respectively, were aspirated through the relevant test tube for $1 \mathrm{~min}$.

The correct perception of ES requires the presence of humid air (Tully and Quinn, 1985), and various voltage-humidity set-ups have been adopted as regular working conditions. In the present study, unless specified, $120 \mathrm{~V}-70 \%$ humidity was used (medium-humidity condition). A $60 \mathrm{~V}-90 \%$ humidity set-up was also used (high-humidity condition).

Statistical significance of the differences between two means, corresponding to mutant and control, were assessed with Student's $t$ test.
Comparisons among multiple means (see Fig. 1) were assessed with one-way ANOVA.

\section{RESULTS}

The olfactory associative conditioning protocol that produces the strongest learning scores consists of the following sequence (Fig. 1A) (Tully and Quinn, 1985). A first odor is presented to a group of flies paired with pulses of ES. After a rest period, a second odor is presented in the absence of shock. To measure the association between the first odor and ES, flies are brought to a choice point from which they have free access to two compartments, each filled with one of the odors previously used during training. Animals, having learned and remembered the odor-ES association, will tend to avoid the corresponding odor.

A major drawback of this procedure is that flies make their olfactory choice after having received strong ES, which might itself induce behavioral changes unrelated to learning and memory, only indirectly affecting learning and memory performances. In particular, because unimpaired olfaction is a prerequisite for the correct interpretation of results in the olfactory associative conditioning protocol, I tested the OA of wild-type and mutant flies after presentation of ES (Fig. $1 B$ ). In this test, a first repellent odor is presented to the flies in association with ES. The flies are then brought to the choice point, where their reactivity to a second repellent odor is measured. This protocol is thus similar to the associative conditioning procedure, but the second odor is not presented during the first phase, whereas the first odor, which has been associated with the shocks, is not presented during the 


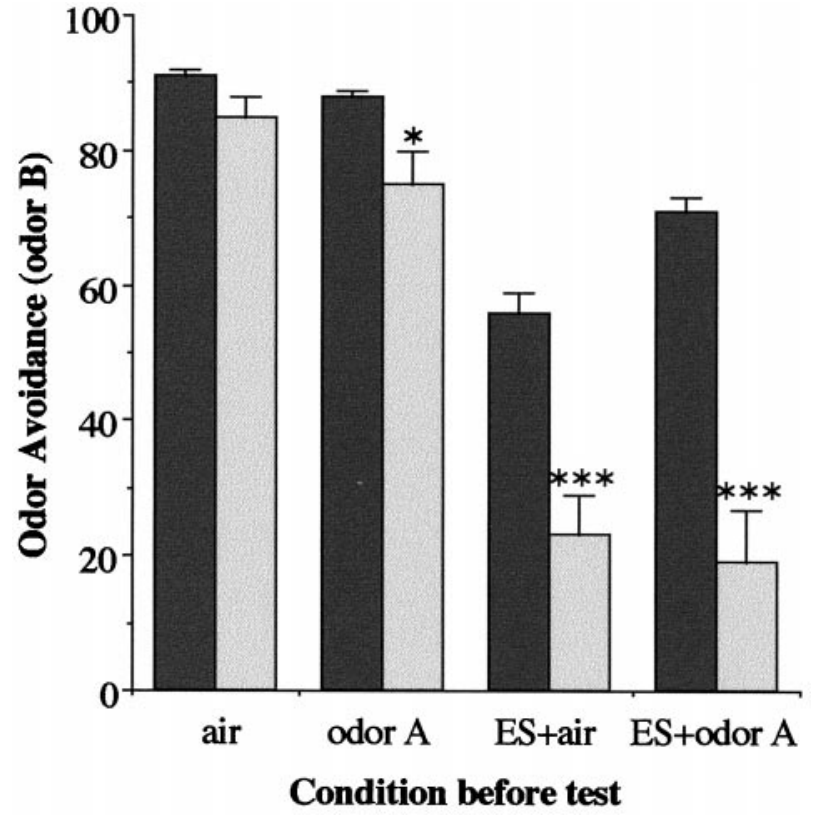

Figure 2. OA of Canton-S (black) and amn (light gray) strains after various experimental conditions. Odor $\mathrm{A}$ is benzaldehyde, and the OA is measured to methylcyclohexanol. No difference is detected between Canton-S and amn after air presentation ( $t$ test; $p=0.054 ; t=2.2)$. After presentation of odor A alone, amn avoidance is marginally reduced ( $t$ test; $\left.{ }^{*} p=0.041 ; t=2.3\right)$, whereas it is strongly reduced after presentation of ES $(E S+$ air $)(t$ test; $* * * p=0.0006 ; t=4.9)$ or ES combined with odor A $(E S+$ odor $A)(t$ test; *** $p=0.0007 ; t=4.8) . n=6$ groups.

test. Such control of the OA of the mutants is more relevant to the associative conditioning protocol than the direct test of naive animals. The fact that the second odor, used to test OA, is novel to the flies at the time of testing precludes interference from phenomena related to multiple presentations, such as habituation.

After presentation of ES combined with the first odor, the mutants $a m n, d n c^{1}, r u t^{1}$, and $r u t^{\mathrm{P} 2080}$ displayed strongly reduced avoidance of a second odor compared with normal flies (Fig. 1B). On the contrary, lio ${ }^{1}$ and lio $^{2}$ behaved in the same way as wild-type flies. Thus, although naive $a m n, d n c$, and rut mutants have been shown in previous studies to react normally to odors (Dudai et al., 1976, 1984; Quinn et al., 1979), their OA is dramatically reduced after presentation of ES, the stimulus normally used for aversive conditioning.

The amn mutant was chosen, together with the reference wild-type stock Canton-S, to analyze in more detail the effect of ES on OA. amn displays an abnormal OA to odor B after presentation of ES, whether ESs are delivered in combination with fresh air or with odor A (Fig. 2). This result indicates that ES presentation is the main cause of the abnormal OA and not preexposure to a first repellent odor. In the case of Canton-S, avoidance of odor B is less efficient when ES is associated with air rather than with odor A (Fig. 2). A plausible explanation for this is that Canton-S flies, having learned the association between the air flow and ES, tend to avoid air during the test. Consequently, to measure any learning-independent effects of ES on OA, it is imperative to present ES associated with odor A, a condition which will not be one of the options in the test.

If $a m n$ displays a strong OA decrease after a $120 \mathrm{~V}$ ES presentation, Canton-S is also affected at this voltage (Fig. 3). This is apparently attributable to the degree of sensitivity, because

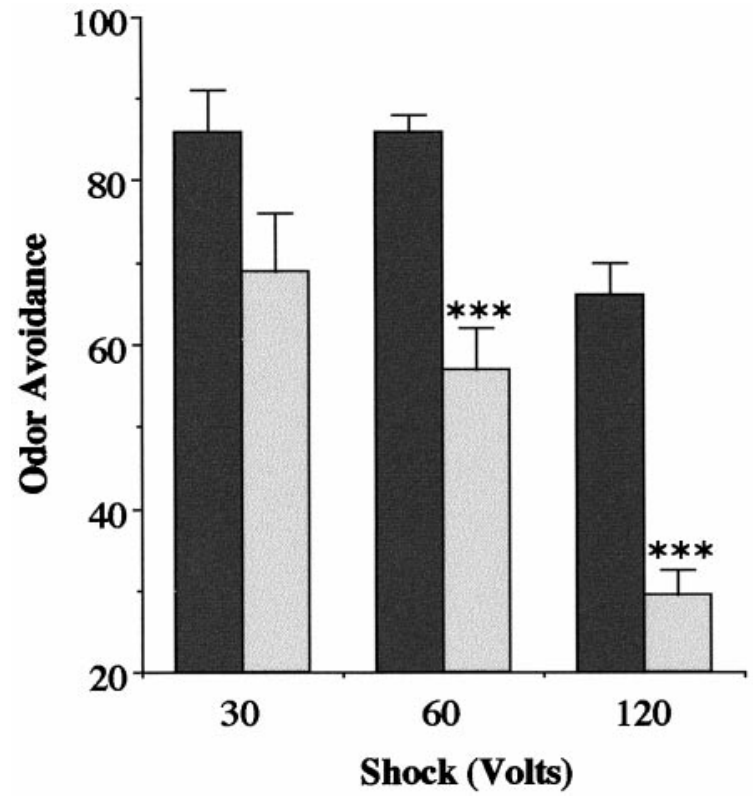

Figure 3. OA after ES of Canton-S (black) and amn (light gray) as a function of ES intensity. No difference is detected at $30 \mathrm{~V}(t$ test; $p=$ $0.072 ; t=2.0)$. A significant difference is detected at $60 \mathrm{~V}(t$ test; $* * * p=$ $0.0005 ; t=5.1)$ and at $120 \mathrm{~V}(t$ test; $* * * p=0.0001 ; t=7.0)$. Odors as in Figure 2. $n=6$ groups.

Canton-S is unaffected at $60 \mathrm{~V}$ (with medium humidity; see Materials and Methods), whereas amn flies are abnormal. Thus, it seems that the threshold for ES perturbation is lower in amn mutants than in normal flies. At this stage, the nature of the physiological changes induced by ES remains to be determined; ES could affect olfaction per se, or it could produce a more general effect on brain activity. Whatever the case, the concern is to understand to what degree the decreased OA affects the performance of the mutants after associative conditioning. In particular, could it account for part of the lasting deficit of amn, which has been interpreted up to now as a memory problem? Indeed, testing OA $1 \mathrm{hr}$ after ES presentation shows no amelioration of the amn defect (Fig. 4), which has only partially recovered at $24 \mathrm{hr}$.

A fundamental issue now is to differentiate learning and memory defects from unrelated deficits. The Dnc phosphodiesterase and the Rut adenylyl cyclase are preferentially expressed in the mushroom bodies, but these enzymes are also found in other parts of the brain (Nighorn et al., 1991; Han et al., 1992). The mushroom body expression might indeed be related to learning and/or memory, whereas the "general" expression might be involved in the nonassociative response described here. To test this hypothesis, I looked at the OA after ES of two $d n c$ deficiencies, which remove different sets of $d n c$ transcripts (Qiu and Davis, 1993). The first deficiency, $D f(1) N^{79 f}$, dramatically decreased Dnc expression in the mushroom bodies. Individuals carrying this deficiency displayed a normal OA after ES (Fig. 5). On the contrary, $D f(1) N^{64 \mathrm{j} 15}$ affected the general Dnc expression, as well as the mushroom body expression. These animals showed a strong decrease of their OA after ES (Fig. 5).

\section{DISCUSSION}

The present results strongly suggest that part of the learning and memory deficits displayed by the mutants $a m n, d n c$, and rut in an associative conditioning protocol could be attributable to effects 


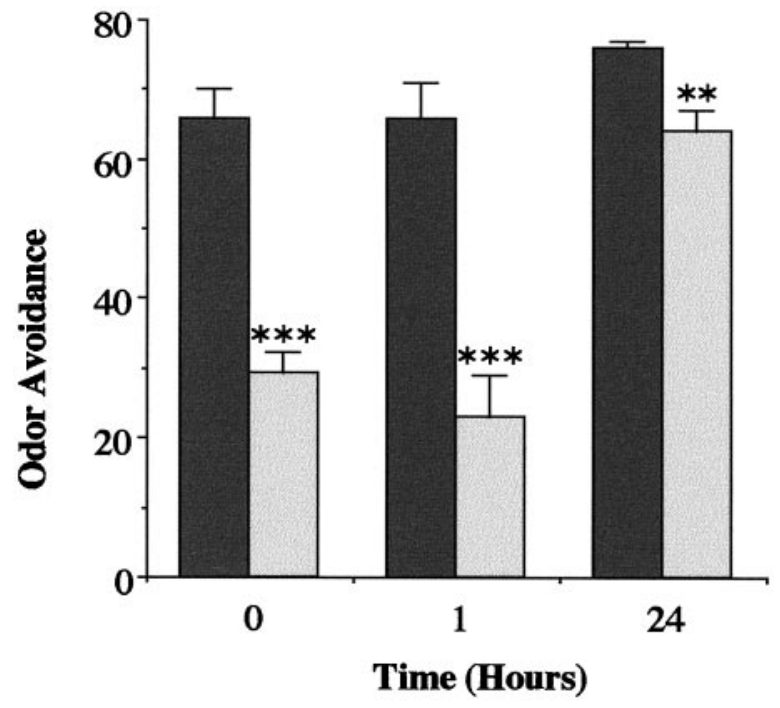

Figure 4. OA of Canton-S (black) and amn (light gray) as a function of the delay between ES delivery and testing. A significant difference is detected at all points: $t=0 \mathrm{hr}, t$ test; ${ }^{* * *} p=0.0001 ; t=7.0 ; t=1 \mathrm{hr}, t$ test; *** $p=0.0003 ; t=5.3 ; t=24 \mathrm{hr}, t$ test; $* * p=0.010 ; t=3.7$. Odors as in Figure 2. $n=6$ groups, except $n=4$ groups at $24 \mathrm{hr}$.

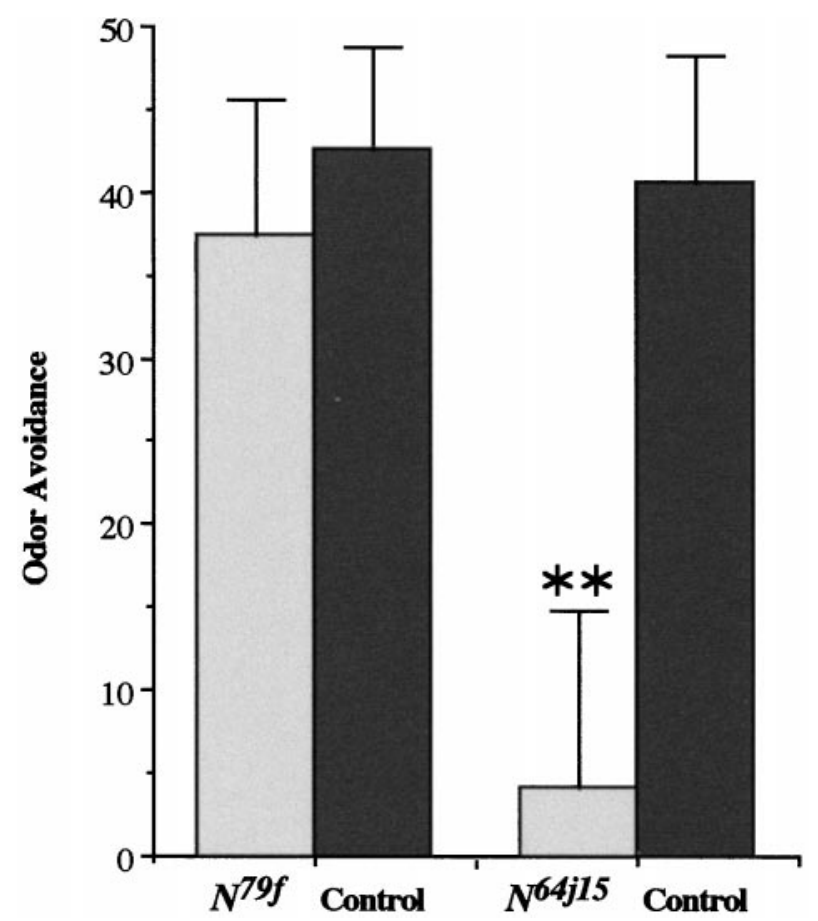

Figure 5. OA after ES of $D f(1) N / w^{+} Y$ males (light gray) and control $C D(1), y w f$ sisters (black). Only $D f(1) N^{64 j 15} / w^{+} Y$ males are affected $(t$ test; $\left.{ }^{* *} p=0.005 ; t=2.77\right)$. Odors as in Figure $1 B . n=12$ groups for $N^{64 j 15} ; n=16$ groups for $N^{79 f}$. The standard condition of $60 \mathrm{~V}$-high humidity was used for this experiment. $w^{\mathrm{a}} D f(1) N^{79 \mathrm{f}} / w^{+} Y$ (Canton-S) males displayed a 90 min memory score of $30.6 \pm 4.3$ compared with $65.1 \pm 5.4$ for $w^{1118} / w^{+} Y$ (Canton-S) control males ( $n=6$ groups; $t$ test; $* * * p=0.0005 ; t=5)$.

of the ES on some aspect of perception in the animals. It is possible that ES alters faculties in the mutants required not only for the test but also for the training phase of associative conditioning, and it is likely that these faculties are progressively deteriorated as the shock pulses are delivered. In summary, an
Table 1. Genetic dissection of $d n c$ behavioral functions ${ }^{a}$

\begin{tabular}{llllll} 
& $\begin{array}{l}\text { Dnc } \\
\text { mushroom } \\
\text { body } \\
\text { expression }\end{array}$ & $\begin{array}{l}\text { Dnc general } \\
\text { brain }\end{array}$ & OA & & Memory \\
Genotype & OAsion & after ES & Learning & $\begin{array}{l}\text { Mem min) } \\
\text { (90 }\end{array}$ \\
\hline Canton-S & + & + & + & + & + \\
$d n c^{1}$ & - & - & - & - & - \\
$D f(1) N^{79 f}$ & $-{ }^{b}$ & + & + & + & - \\
$D f(1) N^{64 j 15}$ & - & - & - & - & -
\end{tabular}

${ }^{a}$ Data reprinted from Qiu and Davis (1993), except for OA.

${ }^{b}$ It should be noted that $D f(1) N^{79 f}$ very strongly decreases Dnc expression in the mushroom bodies but does not eliminate this expression completely (Qiu and Davis, 1993).

altered physiology in the mutants after ES presentation could lead to an apparent defect in associative conditioning because of weaker acquisition during training and/or because of poorer performance during the test.

This work highlights the need for two-step controls in associative learning and memory experiments, especially in which a strong stimulus is used to condition the animals. In particular, the effect of the aversive unconditioned stimulus on the ability of the animals to perceive the conditioned stimulus should be investigated. Thus, although the mutants studied here have been shown to perform poorly under many different associative conditioning protocols (Aceves-Pina and Quinn, 1979; Folkers, 1982; Mariath, 1985), the unconditioned stimulus was generally stressful.

Two strategies can now be adopted to differentiate learning and memory defects from unspecific deficits. First, genetic dissection might reveal that a specific protein isoform and/or expression in a particular subdomain is involved in only one type of behavior. Such an approach was performed successfully with $d n c$ (Table 1) by testing two previously characterized deficiencies (Qiu and Davis, 1993). Thus, a dramatic and specific decrease in Dnc mushroom body product correlates with normal OA after ES. These animals showed a specific memory defect. On the contrary, elimination of the entire brain expression of Dnc leads to a strongly reduced OA after ES. The fact that only the latter animals showed an apparent learning deficit suggests that this might be a secondary consequence of the nonassociative deficit induced by ES. In this hypothesis, the Dnc phosphodiesterase accumulated in the mushroom bodies would be involved specifically in memory formation.

Second, the mutants could be studied under conditions designed to reduce stress to prevent the occurrence of nonspecific defects which might interfere with the conditioning procedure. Thus, it has been seen that $d n c$, which apparently displays a learning defect when conditioned with a negative stimulus (ES), learns normally when a positive stimulus (sugar) is used in association with odors (Tempel et al., 1983). This observation supports the idea that Dnc is specifically required for associative memory but not for associative learning.

\section{REFERENCES}

Aceves-Pina EO, Quinn WG (1979) Learning in normal and mutant Drosophila larvae. Science 15:93-95.

Chen CN, Denome S, Davis RL (1986) Molecular analysis of cDNA clones and the corresponding genomic coding sequences of the Drosophila dunce ${ }^{+}$gene, the structural gene for cAMP phosphodiesterase. Proc Nat Acad Sci USA 83:9313-9317.

Dauwalder B, Davis RL (1995) Conditional rescue of the dunce 
learning/memory and female fertility defects with Drosophila or rat transgenes. J Neurosci 15:3490-3499.

Davis RL (1993) Mushroom bodies and Drosophila learning. Neuron 11:1-14.

de Belle JS, Heisenberg M (1994) Associative odor learning in Drosophila abolished by chemical ablation of mushroom bodies. Science 263:692-695.

Dudai Y, Jan YN, Byers D, Quinn WG, Benzer S (1976) Dunce, a mutant of Drosophila, deficient in learning. Proc Natl Acad Sci USA 5:1684-1688.

Dudai Y, Zvi S, Segel S (1984) A defective conditioned behavior and a defective adenylate cyclase in the Drosophila mutant rutabaga. J Comp Physiol [A] 155:569-576.

Dura JM, Préat T, Tully T (1993) Identification of linotte, a new gene affecting learning and memory in Drosophila melanogaster. J Neurogenet 9:1-14.

Dura JM, Taillebourg E, Préat T (1995) The Drosophila learning and memory gene linotte encodes a putative receptor tyrosine kinase homologous to the human RYK gene product. FEBS Lett 370:250-254.

Erber J, Masuhr T, Menzel R (1980) Localization of short-term memory in the brain of the bee, Apis mellifera. Physiol Entomol 5:343-358.

Feany MB, Quinn WG (1995) A neuropeptide gene defined by the Drosophila memory mutant amnesiac. Science 268:869-873.

Folkers E (1982) Visual learning and memory of Drosophila melanogaster wild type c-s and the mutants dunce, amnesiac, turnip and rutabaga. J Insect Physiol 6:535-539.

Hammer M (1993) An identified neuron mediates the unconditioned stimulus in associative olfactory learning in honeybees. Nature 366:59-63.

Han PL, Levin LR, Reed RR, Davis RL (1992) Preferential expression of the Drosophila rutabaga gene in mushroom bodies, neural centers for learning in insects. Neuron 9:619-627.

Levin LR, Han PL, Hwang PM, Feinstein PG, Davis RL, Reed RR (1992) The Drosophila learning and memory gene rutabaga encodes a $\mathrm{Ca} 2+/$ calmodulin-responsive adenylyl cyclase. Cell 68:479-489.

Mariath HA (1985) Operant conditioning in Drosophila melanogaster wild-type and learning mutants with defects in the cyclic AMP metabolism. J Insect Physiol 10:779-787.

Nighorn AM, Healy MJ, Davis RL (1991) The cyclic AMP phosphodiesterase encoded by the Drosophila dunce gene is concentrated in the mushroom body neuropil. Neuron 6:455-467.

Qiu Y, Davis RL (1993) Genetic dissection of the learning/memory gene dunce of Drosophila melanogaster. Genes Dev 7:1447-1458.

Quinn WG, Harris W, Benzer S (1974) Conditioned behavior in Drosophila melanogaster. Proc Natl Acad Sci USA 71:708-712.

Quinn WG, Sziber PP, Booker R (1979) The Drosophila memory mutant amnesiac. Nature 277:212-214.

Simon AF, Boquet I, Synguélakis M, Préat T (1998) The Drosophila putative kinase Linotte (Derailed) prevents central brain axons from converging on a newly described interhemispheric ring. Mech Dev, in press.

Tempel BL, Bonini N, Dawson DR, Quinn WG (1983) Reward learning in normal and mutant Drosophila. Proc Natl Acad Sci USA 80:1482-1486.

Tully T, Quinn WG (1985) Classical conditioning and retention in normal and mutant Drosophila melanogaster. J Comp Physiol [A] 157:263-277.

Zhong Y, Wu CF (1991) Altered synaptic plasticity in Drosophila memory mutants with a defective cyclic AMP cascade. Science 251:198-200. 